Pacific Journal of Mathematics

FOR EVERY HAUSDORFF SPACE $Y$ THERE EXISTS A
NONTRIVIAL MOORE SPACE ON WHICH ALL CONTINUOUS

FUNCTIONS INTO $Y$ ARE CONSTANT 
FOR EVERY HAUSDORFF SPACE $Y$ THERE EXISTS

A NON-TRIVIAL MOORE SPACE ON WHICH ALL CONTINUOUS FUNCTIONS INTO $Y$

\title{
ARE CONSTANT
}

\section{HaRAld Brandenburg AND AdAm Mysior}

\begin{abstract}
Recently $\mathrm{H}$. Herrlich has raised the question whether there exists a single Moore space $Y$ such that every Moore space can be embedded into some topological power of $Y$. We answer this question negatively by proving the theorem stated in the title.
\end{abstract}

1. Introduction. It was shown independently by N. C. Heldermann [3] and the second named author [8] that there exists a single developable $T_{1}$-space $Y$ such that every developable $T_{1}$-space can be embedded into some topological power of $Y$. In his recent survey article [5] H. Herrlich has raised the problem whether the same is true for Moore spaces. We solve this problem by proving the following:

THEOREM. For every Hausdorff space $Y$ there exists a non-trivial metacompact Moore space on which all continuous functions into $Y$ are constant.

This theorem shows that there does not exist any Hausdorff space $Y$ such that every Moore space can be embedded into some topological power of $Y$, in particular there exists no Moore space with this property.

Another consequence of our theorem is that in the special case that $Y$ is the space of reals, one obtains a metacompact Moore space on which every real-valued continuous function is constant. The first example of a Moore space which admits only constant real-valued continuous functions was found by S. Armentrout [1] based on a non-completely regular Moore space constructed by F. B. Jones [6]. Other examples were given by J. N. Younglove [11] and P. Roy (cited in [7]). Although their spaces are not metacompact they do have other nice properties. On the other hand, we believe that the construction presented here is simpler.

Our theorem should be compared with a result of $\mathrm{H}$. Herrlich, who proved in [4] that for every $T_{1}$-space $Y$ there exists a non-trivial regular space on which all continuous functions into $Y$ are constant. As in this proof, our argument consists of two steps. In $\$ 2$ we construct for every 
Hausdorff space $Y$ a metacompact Moore space $S$ containing two distinct points, $-\infty$ and $+\infty$, such that $f(-\infty)=f(+\infty)$ for every continuous function $f$ from $S$ into $Y$. Using this space and a suitable modification of Herrlich's technique, we complete the proof of the theorem in $\S 3$. The paper ends with some additional remarks. In particular we show that the method introduced in $\$ 2$ can be used to give a simple argument for the first step of the proof of Herrlich's theorem.

The notation we use is standard and can be found in [2]. A topological space is developable if it has a development, i.e. a sequence $\left\{\mathscr{Q}_{n} \mid n \in \mathbf{N}\right\}$ of open covers such that $\left\{\operatorname{St}\left(x, \mathscr{U}_{n}\right) \mid n \in \mathbf{N}\right\}$ is a neighborhood base for every point $x \in X$, where $\operatorname{St}\left(x, \mathcal{Q}_{n}\right)=\cup\left\{U \in \mathcal{Q}_{n} \mid x \in U\right\}$. A regular developable space is called a Moore space. A Moore space is metacompact if it has a development which consists of point-finite open covers. Note that the class of metacompact Moore spaces coincides with the class of regular spaces having a uniform base ([2], 5.4.7).

2. Key lemma. The main step in the proof of our theorem is Lemma 2 below. For its verification we use the following result of Bernstein type, which seems to be a part of the set-theoretical folklore. However, for the sake of completeness, we include a brief argument.

LEMMA 1. Let $\lambda$ be an uncountable cardinal. If $\mathcal{G}$ is a family of subsets of a set $X$ such that $|\mathcal{G}|=\lambda$ and $|G|=\lambda$ for each $G \in \mathcal{G}$, then there exists $a$ denumerable partition $\mathscr{Z}$ of $X$ such that $|G \cap Z|=\lambda$ for each $G \in \mathcal{G}$ and $Z \in \mathcal{Z}$.

Proof. For every $G \in \mathcal{G}$ there exists a partition $\mathscr{F}_{G}$ of $G$ into $\lambda$ sets of cardinality $\lambda$. The set $\mathscr{F}=\bigcup\left\{\mathscr{F}_{G} \mid G \in \mathcal{G}\right\}$ can be written as $\mathscr{F}=\left\{F_{\gamma} \mid 0 \leq\right.$ $\gamma<\lambda\}$. Using transfinite induction one can choose, for every $\gamma<\lambda$, a denumerable set $Z_{\gamma}=\left\{Z_{\gamma, i} \mid i<\omega\right\} \subset F_{\gamma}-\cup\left\{Z_{\alpha} \mid 0 \leq \alpha<\gamma\right\}$. If $Z_{l}=$ $\left\{Z_{\gamma, i} \mid 0 \leq \gamma<\lambda\right\}$ for each $i \geq 2$ and $Z_{1}=X-\cup\left\{Z_{i} \mid i \geq 2\right\}$, then $\mathscr{Z}=$ $\left\{Z_{i} \mid i<\omega\right\}$ is the desired partition of $X$.

Lemma 2. For every Hausdorff space $Y$ there exists a metacompact Moore space $S$ containing two distinct points $-\infty$ and $+\infty$ such that $f(-\infty)=f(+\infty)$ for every continuous function $f$ from $S$ into $Y$.

Proof. Let $Y$ be an arbitrary Hausdorff space and let $\kappa$ be a cardinal such that $|Y| \leq \kappa$ and $\kappa^{\omega}=2^{\kappa}$. Note, for example, that the cardinal $|Y|+2^{\mid Y}+2^{2^{\mid \eta}}+\cdots$ satisfies the hypotheses on $\kappa$ (see [10], Ch. 8.15). 
Moreover, let $X=(X, d)$ be a metric space of weight $\kappa$ such that every non-empty open subset of $X$ has cardinality $2^{\kappa}$, e.g. the countable power of a discrete space of cardinality $\kappa$ with the usual product metric. Denote by $\mathcal{G}$ the collection of all subsets of $X$ which have cardinality $2^{\kappa}$ and are intersections of $\leq \kappa F_{\sigma}$-sets in $X$. Since $\mathcal{G}$ is of cardinality $2^{\kappa}$, it follows from Lemma 1 that there exists a partition $\left\{Z_{l} \mid i \in \mathbf{Z}\right\}$ of $X$ such that $\left|G \cap Z_{l}\right|=2^{\kappa}$ for each integer $i$ and for each $G$ in $\mathcal{G}$.

The space $S$ is obtained by defining the following topology on $\{-\infty\} \cup X \times X \cup\{+\infty\}$, where $-\infty$ and $+\infty$ are two distinct points not contained in $X \times X$. All points in $X \times X$ with distinct coordinates are isolated. For a point $x$ in $Z_{l}$ a basic neighborhood system of $(x, x)$ in $S$ consists of all sets

$$
\begin{aligned}
U_{n}(x, x)= & \left\{(x, z) \mid z \in Z_{l} \cup Z_{i+1}, d(x, z)<1 / n\right\} \\
& \cup\left\{(z, x) \mid z \in Z_{l} \cup Z_{l+1}, d(x, z)<1 / n\right\},
\end{aligned}
$$

where $n \in \mathbf{N}$. Finally, basic neighborhoods of $-\infty$ and $+\infty$ are of the form

$$
U_{n}(-\infty)=\{-\infty\} \cup \cup\left\{U_{n}(x, x) \mid x \in \cup\left\{Z_{i} \mid i \leq-n\right\}\right\}
$$

and

$$
U_{n}(+\infty)=\{+\infty\} \cup \cup\left\{U_{n}(x, x) \mid x \in \cup\left\{Z_{l} \mid i \geq n\right\}\right\},
$$

respectively. Since basic neighborhoods of points from $X \times X$ are clopen in $S$ and $\mathrm{cl}_{S} U_{n+1}(-\infty) \subset U_{n}(-\infty), \mathrm{cl}_{S} U_{n+1}(+\infty) \subset U_{n}(+\infty)$ for each $n \in \mathbf{N}$, the space $S$ is regular. Moreover, $S$ is a metacompact Moore space - the open covers

$$
\begin{aligned}
\mathscr{Q}_{n}= & \left\{U_{n}(-\infty), U_{n}(+\infty)\right\} \cup\left\{U_{n}(x, x) \mid x \in X\right\} \\
& \cup\left\{\{(y, z)\} \mid(y, z) \notin \cup\left\{U_{n}(x, x) \mid x \in X\right\}\right\}
\end{aligned}
$$

are point-finite and form a development of $S$.

Now let $f$ be an arbitrary continuous function from $S$ into $Y$. To prove that $f(-\infty)=f(+\infty)$ it suffices to show that $f(+\infty) \in V$ for every open neighborhood $V$ of $f(-\infty)$ in $Y$. To this end consider an arbitrary open set $V$ in $Y$ containing $f(-\infty)$. For each $y \in Y$ denote by $A(y)$ the set of all points $x \in X$ such that $f(x, x)=y$. By the continuity of $f$ there exists an $i_{0} \in \mathbf{Z}$ such that $\{A(y) \mid y \in V\}$ covers $Z_{i_{0}}$. Consequently, since $|V| \leq \kappa$ and $\left|Z_{l_{0}}\right|=2^{\kappa}$, there must exist a $y_{0} \in V$ such that $\left|A\left(y_{0}\right) \cap Z_{\iota_{0}}\right|=$ $2^{\kappa}$. We claim that $f(+\infty)=y_{0}$. Note that this is proved once we have 
shown that every neighborhood of $+\infty$ meets $f^{-1}\left(y_{0}\right)$, which, however, is an immediate consequence of the following:

Claim. $\left|A\left(y_{0}\right) \cap Z_{i}\right|=2^{\kappa}$ for every $i \geq i_{0}$.

To prove the claim we proceed by induction. Assume $i \geq i_{0}$ and $\left|A\left(y_{0}\right) \cap Z_{i}\right|=2^{\kappa}$. To see that $\left|A\left(y_{0}\right) \cap Z_{i+1}\right|=2^{\kappa}$ it suffices to construct a set $G$ in $\mathcal{G}$ such that $G \cap Z_{i+1} \subset A\left(y_{0}\right) \cap Z_{l+1}$. For this purpose we define for every point $y$ in $Y$ distinct from $y_{0}$ an $F_{\sigma}$-set $F(y)$ in $X$ containing $A\left(y_{0}\right) \cap Z_{l}$ and disjoint from $A(y) \cap Z_{i+1}$. Observe that $G=$ $\cap\left\{F(y) \mid y \in Y, y \neq y_{0}\right\}$ then has the desired properties.

Let $y$ be an arbitrary point in $Y$ distinct from $y_{0}$. There exists an open neighborhood $W$ of $y_{0}$ in $Y$ which does not contain $y$ in its closure. For each $n \in \mathbf{N}$, denote by $A_{n}$ the set of all points $x \in A\left(y_{0}\right) \cap Z_{l}$ such that $f\left(U_{n}(x, x)\right) \subset W$. Since $F(y)=\bigcup\left\{\mathrm{cl}_{X} A_{n} \mid n \in \mathbf{N}\right\}$ is an $F_{\sigma}$-set in $X$ containing $A\left(y_{0}\right) \cap Z_{i}$, it only remains to prove that $\mathrm{cl}_{X} A_{n}$ and $A(y) \cap Z_{i+1}$ are disjoint for each $n \in \mathbf{N}$. To this end consider an arbitrary $n \in \mathbf{N}$ and a point $z \in A(y) \cap Z_{i+1}$. Since $f(z, z)$ does not belong to the closure of $W$ in $Y$, there exists an $m \geq n$ such that $f\left(U_{m}(z, z)\right) \cap W=\varnothing$. It follows that $d(x, z) \geq 1 / m$ for each $x \in A_{n}$, for otherwise there would exist an $x \in A_{n}$ such that $U_{m}(x, x) \cap U_{m}(z, z) \neq \varnothing$, which is impossible. Hence $z \notin \mathrm{cl}_{X} A_{n}$, which completes the proof.

3. Proof of the theorem. Let $Y$ be an arbitrary Hausdorff space. By virtue of Lemma 2 there exists a metacompact Moore space $S$ containing two distinct points, $-\infty$ and $+\infty$ such that $f(-\infty)=f(+\infty)$ for every continuous function $f$ from $S$ into $Y$. Using an ingenious construction of H. Herrlich [4], one can now obtain from $S$ a regular space $X$ on which every continuous function into $Y$ is constant. However, since we want the resulting space to be a metacompact Moore space, we have to modify this construction slightly.

Our first aim is to construct for every Moore space $M$ a Moore space $S(M)$ containing $M$ as a closed subspace such that every continuous function from $S(M)$ into $Y$ is constant on $M$. Additionally $S(M)$ will be metacompact if and only if $M$ is metacompact.

Let $M$ be an arbitrary Moore space and let $\left\{\mathcal{V}_{n} \mid n \in \mathbf{N}\right\}$ be a development of $M$ such that $\mathscr{V}_{n+1}$ refines $\mathscr{V}_{n}$ for each $n \in \mathbf{N}$. The underlying set of $S(M)$ is $\{p\} \cup\left(S^{\prime} \times M\right) \cup M$, where $S^{\prime}=S$ $\{-\infty,+\infty\}$ and $p$ is a point neither contained in $S^{\prime} \times M$ nor in $M$. For each $n \in \mathbf{N}$ we define a cover $S\left(\mathscr{V}_{n}\right)$ of $S(M)$ as follows. Let $\left\{\mathscr{U}_{n} \mid n \in \mathbf{N}\right\}$ 
be a development of $S$ such that $\mathscr{U}_{n+1}$ refines $\mathcal{U}_{n}$ and $\mathscr{U}_{n}$ is point-finite for each $n \in \mathbf{N}$. Moreover let $\left\{U_{n}^{-} \mid n \in \mathbf{N}\right\}$ and $\left\{U_{n}^{+} \mid n \in \mathbf{N}\right\}$ be sequences of open subsets of $S^{\prime}$ such that $\left\{U_{n}^{-} \cup\{-\infty\} \mid n \in \mathbf{N}\right\}$ and $\left\{U_{n}^{+} \cup\{+\infty\} \mid n \in \mathbf{N}\right\}$ are neighborhood bases in $S$ of $-\infty$ and $+\infty$, respectively, and $\mathrm{cl}_{S^{\prime}} U_{n+1}^{-} \subset U_{n}^{-}, \mathrm{cl}_{S^{\prime}} U_{n+1}^{+} \subset U_{n}^{+}$for each $n \in \mathbf{N}$. Then $S\left(\mathscr{V}_{n}\right)$ consists of $\{p\} \cup\left(U_{n} \times M\right)$, all sets $U \times\{z\}$, where $U \in \mathcal{Q}_{n}$ and $z \in M$, and all sets $S(V)=\left(U_{n} \times V\right) \cup V$, where $V \in \mathscr{V}_{n}$. One can easily check that taking $\cup\left\{S\left(\mathscr{V}_{n}\right) \mid n \in \mathbf{N}\right\}$ as a base yields a regular space $S(M)$ for which $\left\{S\left(\mathscr{V}_{n}\right) \mid n \in \mathbf{N}\right\}$ is a development. Obviously, $M$ is contained as a closed subspace in $S(M)$. For further reference we note some additional properties of $S(M)$ :

(i) $S(V) \cap M=V$ for each $V \in \mathcal{V}_{n}$;

(ii) $S(V) \cap S(W)=\varnothing$ whenever $V, W \in \cup\left\{\mathscr{V}_{n} \mid n \in \mathbf{N}\right\}$ and $V \cap$ $W=\varnothing$;

(iii) $S(W) \subset S(V)$ whenever $V, W \in \cup\left\{\mathscr{V}_{n} \mid n \in \mathbf{N}\right\}$ and $W \subset V$;

(iv) $\operatorname{cl}_{S(M)} S(W) \subset S(V)$ whenever $\mathrm{cl}_{M} W \subset V, W \in \mathcal{V}_{m}, V \in \mathcal{V}_{n}$ and $n<m$;

(v) $S\left(\mathscr{V}_{n+1}\right)$ refines $S\left(\mathcal{V}_{n}\right)$ for each $n \in \mathbf{N}$;

(vi) $S\left(\mathscr{V}_{n}\right)$ is point-finite if and only if $\mathscr{V}_{n}$ is point-finite.

Note that the topology of $S(M)$ is defined in such a way that for every $z \in M$ there is an obvious homeomorphism from $S$ onto the subspace $\{p\} \cup\left(S^{\prime} \times\{z\}\right) \cup\{z\}$ of $S(M)$ mapping $-\infty$ onto $p$ and $+\infty$ onto $z$. Therefore $f(z)=f(p)$ for every continuous function $f$ from $S(M)$ into $Y$, i.e. every such function is constant on $M$.

Starting with an arbitrary Moore space $M_{1}$ having a development $\left\{\mathcal{V}_{n}^{1} \mid n \in \mathbf{N}\right\}$ with every $\mathcal{V}_{n}^{1}$ point-finite and $\mathcal{V}_{n+1}^{1}$ refining $\mathcal{V}_{n}^{1}$, we can now define a sequence $M_{1} \subset M_{2} \subset M_{3} \subset \cdots$ of Moore spaces according to the rule $M_{k+1}=S\left(M_{k}\right)$. In the following we consider for every $M_{k}$ the development $\left\{\mathcal{V}_{n}^{k} \mid n \in \mathbf{N}\right\}$ which is inductively obtained from $\left\{\mathcal{V}_{n}^{1} \mid n \in\right.$ $\mathbf{N}\}$ by $\mathfrak{V}_{n}^{k+1}=S\left(\mathscr{V}_{n}^{k}\right)$. Our aim is to define on $X=\cup\left\{M_{k} \mid k \in \mathbf{N}\right\}$ a topology which turns $X$ into a metacompact Moore space on which every continuous function into $Y$ is constant. For this purpose we introduce the following notation.

If $V \in \mathscr{V}_{n}^{k}$ for some $n, k \in \mathbf{N}$ define $T(V)=\cup\left\{S^{i}(V) \mid i \geq 0\right\}$, where $S^{0}(V)=V, S^{1}(V)=S(V), S^{2}(V)=S(S(V)), \ldots$ It can be easily seen that for each $n \in \mathbf{N}$ the collection $\mathscr{V}_{n}=\left\{T(V) \mid V \in \cup\left\{\mathscr{V}_{n}{ }^{k} \mid k \in\right.\right.$ $\mathbf{N}$ \}) is a cover of $X$. From (v) it follows that $\mathscr{V}_{n+1}$ refines $\mathscr{V}_{n}$ for each $n \in \mathbf{N}$. For each $x \in X$ denote by $k(x)$ the smallest $k \in \mathbf{N}$ such that $x \in M_{k}$. The crucial property of the covers $\mathscr{V}_{n}$ is the following:

(vii) If $T(V) \in \mathscr{V}_{n}$ and $x \in T(V)$, then there exists a $W \in \mathscr{V}_{n}^{k(x)}$ containing $x$ such that $T(V)=T(W)$. 
It can be easily verified using (i) above. As an immediate consequence of (vii) one obtains

(viii) $\operatorname{St}\left(x, \mathscr{V}_{n}\right)=\cup\left\{T(V) \mid V \in \mathfrak{V}_{n}^{k(x)}, x \in V\right\}$ for each $x \in X$ and $n \in \mathbf{N}$.

Using (ii), (iii) and (viii) it is not difficult to check that $\cup\left\{\mathscr{V}_{n} \mid n \in \mathbf{N}\right\}$ is a base for a topology which turns $X$ into a developable $T_{1}$-space. Finally it follows from (iv) that $X$ is a Moore space which is metacompact because of (vi) and (vii).

Now let $f$ be an arbitrary continuous function from $X$ into $Y$. To see that $f$ is constant consider two distinct points $x$ and $x^{\prime}$ in $X$. There exists a $k \in \mathbf{N}$ such that $x$ and $x^{\prime}$ are both contained in $M_{k}$. Since it follows from (i) that $M_{k+1}$ is a subspace of $X$, the restriction of $f$ to $M_{k+1}$ is a continuous function from $M_{k+1}$ into $Y$ which is constant on $M_{k}$ because $M_{k+1}=S\left(M_{k}\right)$. Hence $f(x)=f\left(x^{\prime}\right)$, which completes the proof.

4. Remarks. 1. For every non-empty class \& of topological spaces there exists a smallest class $E H(\mathbb{E})$ of topological spaces which contains \& and is closed with respect to the formation of arbitrary products and subspaces. $E H(\mathbb{E})$ is called the epireflective hull of \&. It consists of all those spaces which can be embedded into products of spaces belonging to E. A class $\mathfrak{U}$ of topological spaces is called simple if there exists a single space $E$ such that $\mathfrak{A}=E H(\{E\})$. Originally Herrlich's question, mentioned in the introduction, was stated in these terms, namely, whether the epireflective hull of the class of all Moore spaces is simple (see [5], Problem 21). Our theorem implies that any class of Hausdorff spaces which contains all metacompact Moore spaces is not simple.

2. A weaker notion than simplicity is that of semi-simplicity of a class of spaces: A class $\mathfrak{A}$ is called semi-simple (e.g. see [5], Ch. 3.2.8) if there exists a minimal class $\mathbb{E}$ of spaces such that $\mathfrak{A}=E H(\mathbb{E})$, where minimality of $\&$ means that $E H(\&-\{E\}) \neq \mathfrak{A}$ for every space $E$ in $\&$. In view of the preceding remark one might wonder whether the epireflective hull of all Moore spaces is semi-simple. We give a brief argument which shows that it is not.

Let $\mathfrak{U}$ be the epireflective hull of all Moore spaces and consider a class $\&$ of spaces such that $\mathfrak{A}=E H(\mathbb{E})$. We claim that $\mathfrak{A}=E H(\mathbb{E}-\{E\})$ for each $E \in \mathbb{E}$. To this end consider an arbitrary $E \in \mathbb{E}$. There exists a family $\left\{M_{i} \mid i \in I\right\}$ of Moore spaces such that $E$ can be embedded into the product of this family. The disjoint union $M$ of all spaces $M_{i}$ is a Moore space such that $E \in E H(\{M\})$. Therefore it suffices to prove that $M \in$ $E H(\&-\{E\})$. From the proof of our theorem it follows that there exists 
a Moore space $X$ containing $M$ as a subspace such that all continuous functions from $X$ into $E$ are constant. It follows that $X \in E H(\&-\{E\})$ and, consequently, that $M \in E H(\&-\{E\})$.

3. It is worth mentioning that such a familiar $T_{1}$-space like the reals with co-finite topology has the property that every Moore space, in fact every developable $T_{1}$-space, can be embedded into some of its powers. To verify this fact it suffices to recall that every developable $T_{1}$-space can be embedded into a product of second countable developable $T_{1}$-spaces $[3,8]$, and that every $T_{1}$-space of cardinality $\leq c$ can be embedded into some power of the reals with co-finite topology (see [2], Problem 2.7.8(b)).

4. We like to indicate how the construction used to prove Lemma 2 can be modified to obtain a simple proof of the theorem of $\mathrm{H}$. Herrlich and A. Ramer (see [2], Problems 2.7.17 and 2.7.18) that for every $T_{1}$-space $Y$ there exists a regular space $S$ containing two distinct points $-\infty$ and $+\infty$ such that $f(-\infty)=f(+\infty)$ for every continuous function from $S$ into $Y$.

In fact, if $\kappa$ is an infinite cardinal such that $|Y| \leq \kappa$, let $\left\{Z_{i} \mid i \in \mathbf{Z}\right\}$ be a family of disjoint sets of cardinality $\kappa^{+}$and consider the set $S=\{-\infty\}$ $\cup X \times X \cup\{+\infty\}$, where $X=\cup\left\{Z_{l} \mid i \in \mathbf{Z}\right\}$. A regular topology on $S$ is defined as follows. All points in $X \times X$ with distinct coordinates are isolated. For a point $x \in Z_{l}$ a basic neighborhood system of $(x, x)$ in $S$ consists of all sets

$$
U_{F}(x, x)=\{(x, x)\} \cup\left(\left(\{x\} \times X \cup Z_{i+1} \times\{x\}\right)-F\right),
$$

where $F$ is an arbitrary finite subset of $X \times X$. Finally, basic neighborhoods of $-\infty$ and $+\infty$ of the form

$$
\begin{aligned}
U_{n}(-\infty)= & \{-\infty\} \\
& \cup \cup\left\{U_{F}(x, x) \mid x \in \cup\left\{Z_{i} \mid i \leq-n\right\}, F \subset X \times X \text { finite }\right\}
\end{aligned}
$$

and

$$
\begin{aligned}
U_{n}(+\infty)= & \{+\infty\} \\
& \cup \cup\left\{U_{F}(x, x) \mid x \in \cup\left\{Z_{i} \mid i \geq n\right\}, F \subset X \times X \text { finite }\right\},
\end{aligned}
$$

respectively. If $f$ is a continuous function from $S$ into $Y$ and $V$ is a neighbourhood of $f(-\infty)$, there exists an $i_{0}$ in $\mathbf{Z}$ such that $f$ maps $\left\{(x, x) \mid x \in Z_{i_{0}}\right\}$ into $V$. Since $|V| \leq \kappa$ and $\left|Z_{l_{0}}\right|=\kappa^{+}$, there exists a point $y_{0}$ in $V$ and a denumerable subset $A_{\iota_{0}}$ of $Z_{i_{0}}$ such that $\left\{(x, x) \mid x \in A_{i_{0}}\right\} \subset$ $f^{-1}\left(y_{0}\right)$. Inductively one can now show that $f^{-1}\left(y_{0}\right)$ contains infinitely many points of $\left\{(x, x) \mid x \in A_{l}\right\}$ for every $i \geq i_{0}$. We omit the details, for the argument is similar to that used in [9]. It follows that $f(+\infty)=y_{0} \in V$, consequently $f(-\infty)=f(+\infty)$. 


\section{REFERENCES}

[1] S. Armentrout, A Moore space on which every real-valued continuous function is constant, Proc. Amer. Math. Soc., 12 (1961), 106-109.

[2] R. Engelking, General Topology, Polish Scientific Publishers, Warsaw, 1977.

[3] N. C. Heldermann, The category of D-completely regular spaces is simple, Trans. Amer. Math. Soc., 262 (1980), 437-446.

[4] H. Herrlich, Wann sind alle stetigen Abbildungen in Y konstant?, Math. Zeitschr., 90 (1965), 152-154.

[5] Categorical Topology 1971-1981, in: General Topology and its Relations to Modern Analysis and Algebra V (Proc. of the Fifth Prague Topological Symposium, Prague 1981), ed. by J. Novák, Heldermann Verlag, Berlin (1983).

[6] F. B. Jones, Moore spaces and uniform spaces, Proc. Amer. Math. Soc., 9 (1958), 483-486.

[7] Constructing non-completely regular spaces, in: Topology and its Applications (Proc. of the Topology Conference Budva 1972), ed. by D. Kurepa, Beograd, 1973, $132-135$.

[8] A. Mysior, Two remarks on D-regular spaces, Glasnik Mat., 15 (1980), 153-156.

[9] $\quad$ A regular space which is not completely regular, Proc. Amer. Math. Soc., 81 (1981), 652-653.

[10] W. Sierpin'ski, Cardinal and Ordinal Numbers, Polish Scientific Publishers, Warsaw, 1965.

[11] J. N. Younglove, A locally connected, complete Moore space on which every real-valued continuous function is constant, Proc. Amer. Math. Soc., 20 (1969), 527-530.

Received February 8, 1982 and in revised form July 29, 1982.

INSTITUT FUR MATHEMATIK I

FREIE UNIVERSITÄT BERLIN

D-1000 BERLIN 33

Federal Republic of Germany

AND

INSTYTUT MATEMATYKI

UNIWERSYTET GDAŃSKI

80-952 GDAŃSK

POLAND 


\section{PACIFIC JOURNAL OF MATHEMATICS}

EDITORS

Donald BabBITT (Managing Editor)

University of California

Los Angeles, CA 90024

Hugo Rossi

University of Utah

Salt Lake City, UT 84112

C. C. Moore and Arthur Ogus

University of California

Berkeley, CA 94720
J. DugunduI

Department of Mathematics

University of Southern California

Los Angeles, CA 90089-1113

R. FINN and H. SAMELSON

Stanford University

Stanford, CA 94305

\section{ASSOCIATE EDITORS}
R. ARENS
E. F. BECKENBACH
B. H. NeumanN
F. WolF
K. YosHIDA (1906-1982)

\section{SUPPORTING INSTITUTIONS}

UNIVERSITY OF ARIZONA

UNIVERSITY OF BRITISH COLUMBIA

CALIFORNIA INSTITUTE OF TECHNOLOGY

UNIVERSITY OF CALIFORNIA

MONTANA STATE UNIVERSITY

UNIVERSITY OF NEVADA. RENO

NEW MEXICO STATE UNIVERSITY

OREGON STATE UNIVERSITY
UNIVERSITY OF OREGON

UNIVERSITY OF SOUTHERN CALIFORNIA

STANFORD UNIVERSITY

UNIVERSITY OF HAWAII

UNIVERSITY OF TOKYO

UNIVERSITY OF UTAH

WASHINGTON STATE UNIVERSITY

UNIVERSITY OF WASHINGTON 


\section{Pacific Journal of Mathematics}

\section{Vol. 111, No. $1 \quad$ November, 1984}

Harald Brandenburg and Adam Stefan Mysior, For every Hausdorff

space $Y$ there exists a nontrivial Moore space on which all continuous

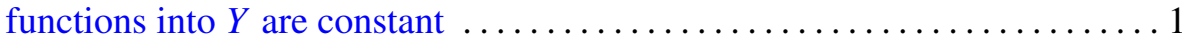

Henry Dappa, A Marcinkiewicz criterion for $L^{p}$-multipliers $\ldots \ldots \ldots \ldots \ldots 9$

P. H. Doyle, III and John Gilbert Hocking, Bijectively related spaces. I.

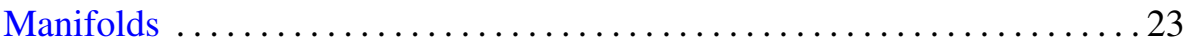

Joel Hass, Complete area minimizing minimal surfaces which are not totally

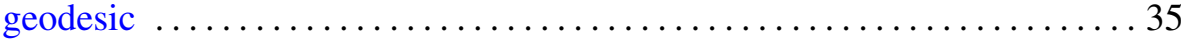

Aarno Hohti, On Ginsburg-Isbell derivatives and ranks of metric spaces .... 39

Richard Howard Hudson, Diophantine determinations of $3^{(p-1) / 8}$ and $5^{(p-1) / 4}$

A. F. Izé and A. Ventura, Asymptotic behavior of a perturbed neutral functional-differential equation related to the solution of the unperturbed

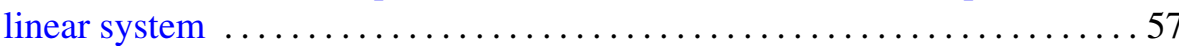

Palle E. T. Jorgensen, Spectral representations of unbounded nonlinear

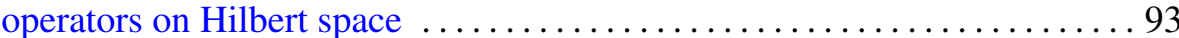

Darrell Conley Kent and Gary Douglas Richardson, Cauchy spaces with

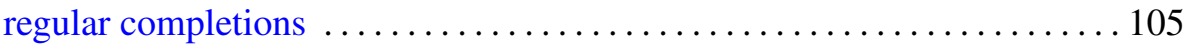

Mark Mahowald, An addendum to: "bo-resolutions" ................ 117

Stuart Wayne Margolis and Jean-Eric Pin, Minimal noncommutative varieties and power varieties

Carla Massaza and Alfio Ragusa, Some conditions on the homology groups of the Koszul complex

Vicente Miquel Molina, Some examples of Riemannian almost-product manifolds

Roderic Murufas, Inverse spectral problems for certain differential operators

Ulrich Oertel, Closed incompressible surfaces in complements of star links

Katsuro Sakai, A characterization of local equiconnectedness

William Victor Smith and Don Harrell Tucker, Weak integral convergence theorems and operator measures 